\title{
Design and Characteristic Analysis of Multicarrier Chaotic Phase Coded Radar Pulse Train Signal
}

\author{
Qiongdan Huang, Yong Li, Yaoping Zeng, and Yinjuan Fu \\ School of Electronics and Information, Northwestern Polytechnical University, Xian 710072, China \\ Correspondence should be addressed to Yong Li; ruikel@nwpu.edu.cn
}

Received 16 January 2014; Revised 24 March 2014; Accepted 7 April 2014; Published 12 May 2014

Academic Editor: J.S. Mandeep

Copyright ( 2014 Qiongdan Huang et al. This is an open access article distributed under the Creative Commons Attribution License, which permits unrestricted use, distribution, and reproduction in any medium, provided the original work is properly cited.

By introducing phase code into multicarrier orthogonal frequency division multiplex signal, the multicarrier phase coded (MCPC) radar signal possesses a good spectrum utilization rate and can achieve a good combination of narrowband and wideband processing. Radar pulse train signal not only reserves the high range resolution of monopulse signal, but also has the same velocity resolution performance as continuous wave signal does. In this study, we use the chaotic biphase code generated by Chebyshev mapping to conduct a phase modulation on MCPC pulse train so as to design two different types of multicarrier chaotic phase coded pulse train signal. The ambiguity functions of the two pulse train signals are compared with that of $\mathrm{P} 4$ code MCPC pulse train. In addition, we analyze the influences of subcarrier number, phase-modulated bit number, and period number on the pulse train's autocorrelation performance. The low probability of intercept (LPI) performance of the two signals is also discussed. Simulation results show that the designed pulse train signals have a thumbtack ambiguity function, a periodic autocorrelation side lobe lower than P4 code MCPC pulse train, and excellent LPI performance, as well as the feature of waveform diversity.

\section{Introduction}

The MCPC signal based on OFDM technique [1] is a new wideband radar signal which has attracted much attention recently. It has a flexible structure, a thumbtack ambiguity function. This signal not only has the advantages of narrowband radar but also can synthesize the wideband signal with orthogonal narrowband signals so as to achieve multichannel separation and quick processing. Starting from OFDM signal, Levanon and Mozeson [2] analyzed the structure, spectrum, ambiguity function, autocorrelation, and power spectrum of monopulse, continuous wave, and pulse train of MCPC signal. Besides, the methods for decreasing the peak-to-mean envelope power ratio (PMEPR) of these signals are elaborated. Studies were performed on the waveform design, pulse compression, target detection, and imaging of MCPC signal in [3]. Reference [4] introduced a method of least squares for the allocation of a proper phase to obtain desired ambiguity function. In literature [5], the features of MCPC signal and frequency stepped signal were combined to propose the multicarrier phase coded frequency stepped radar signal, which has a high range resolution yet has the defect of range-Doppler coupling.

In order to extract Doppler information, monopulse needs to be accumulated to obtain the pulse train signal. The pulse train signal reserves the high range resolution of monopulse signal while maintaining the velocity resolution performance of continuous wave signal [6]. However, the traditional pulse train signal has a poor performance in measuring remote and high speed targets because of range or velocity ambiguity, and the antijamming performance is also low. MCPC signal has a flexible structure, and different pulse train signals can be obtained using different phase coding methods. Studying the MCPC pulse train signal with complex modulation can improve the detection performance and LPI performance of radar.

Like the noise signal, the chaotic signal exhibits continuous power spectrum, initial-value sensitivity, ergodicity, and aperiodicity, and therefore chaotic signal possesses good range and velocity resolutions, thumbtack ambiguity 
TABLE 1: Classification of MCPC pulse train signal.

\begin{tabular}{lcccc}
\hline & IIS MCPC & INS MCPC & NIS MCPC & NNS MCPC \\
\hline Phase code of each pulse & Identical & Identical & Nonidentical & Nonidentical \\
Phase code of each subcarrier & Identical Sequence & Nonidentical Sequence & Identical Sequence & Nonidentical Sequence \\
\hline
\end{tabular}

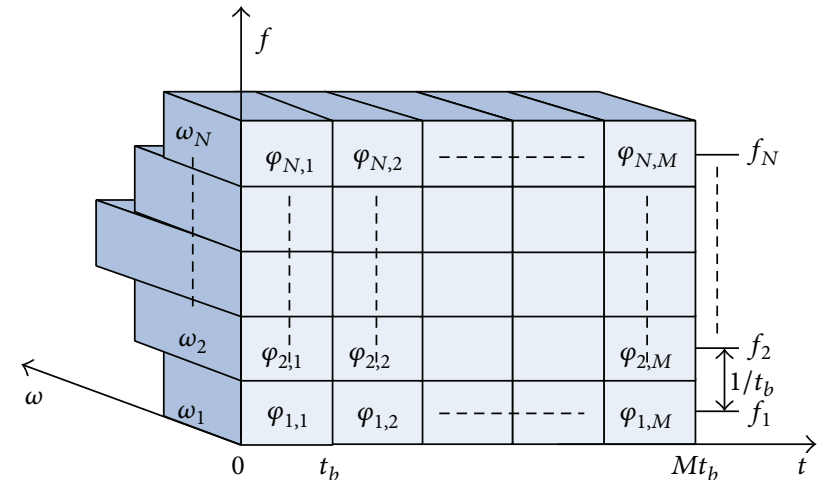

FIGURE 1: Structure of MCPC signal.

function, excellent antijamming ability, and electromagnetic compatibility. Thus, chaotic signal avoids the problem of range ambiguity. Moreover, it overcomes many shortcomings of the noise signal, such as the difficulties of generation, replication, and application. These properties of chaotic signal conform to the low probability of intercept (LPI) and antijamming requirements for modern radar.

In this study, the multicarrier chaotic phase coded pulse train signal is designed using the chaotic biphase code for phase modulation on MCPC pulse train by different modes. The ambiguity function as well as the autocorrelation performance of the signal is simulated, and the LPI performance is also analyzed.

\section{Structure of MCPC Pulse Train Signal}

The complex envelope of the monopulse MCPC signal is given by

$$
\begin{aligned}
f(t)=\sum_{n=1}^{N} \sum_{m=1}^{M} \omega_{n} a_{n, m} s\left[t-(m-1) t_{b}\right] \\
\times \exp (j 2 \pi(n-1) \Delta f t),
\end{aligned}
$$

where $N$ is the subcarrier number and $M$ is the phasemodulated bits. Consider $a_{n, m}=e^{j \varphi_{n, m}}$, where $\varphi_{n, m}$ is the $m$ th phase element of the $n$th subcarrier. One has $s(t) \equiv 1$ for $0 \leq$ $t<t_{b}$ and zero elsewhere. The frequency difference between two adjacent subcarriers $\Delta f$ is set equal to the inverse of the bit duration $t_{b}$ yielding orthogonal frequency division multiplexing. $\omega_{n}=\left|\omega_{n}\right| e^{j \theta_{n}}\left(\sum_{n=0}^{N-1}\left|\omega_{n}\right|^{2}=1\right)$ is the complex weight associated with the $n$th subcarrier, and $\left|\omega_{n}\right|$ is the frequency weighted amplitude. $\theta_{n}$ is the frequency weighted phase, also known as the initial phase. The structure of the MCPC signal is illustrated schematically in Figure 1.
The schematic diagram shows that the MCPC signal consists of $N$ sequences transmitted simultaneously on $N$ subcarriers. Each sequence contains $M$ phase-modulated bits.

The general expression for the MCPC pulse train complex envelope $f_{T_{r}}(t)$ is

$$
\begin{aligned}
& f_{\operatorname{Tr}}(t) \\
& =\sum_{p=1}^{P} \sum_{n=1}^{N} \sum_{m=1}^{M} w_{n} a_{n, m, p} s\left[t-(m-1) t_{b}-(p-1) T_{r}\right] \\
& \quad \times \exp \left[j 2 \pi(n-1)\left(t-(p-1) T_{r}\right) \Delta f\right],
\end{aligned}
$$

where $P$ is the period number and $T_{r}$ is pulse repetition interval. Consider $a_{n, m, p}=e^{j \varphi_{n, m, p}}$, where $\varphi_{n, m, p}$ is the $m$ th phase element of the $n$th sequence in $p$ th pulse. The rest of the parameters are the same as those of monopulse MCPC signal mentioned above.

\section{Design of Multicarrier Chaotic Phase Coded Radar Pulse Train Signal}

Chaos is considered as a phenomenon of random behavior in certainty nonlinear dynamical system [7]. Chaotic mapping can generate plenty of signals [8] whose rules are difficult to grasp by the interference side. Considering the balance, correlation, and the flatness of power spectrum in band, this study uses Chebyshev mapping [9] to generate the biphase coded sequence.

Chebyshev mapping is defined as

$$
x_{n+1}=\cos \left(k \arccos \left(x_{n}\right)\right) \text {, }
$$

where $x_{0} \in(-1,1)$ and Chebyshev mapping order $k=4$.

The chaotic signal generated by chaotic maps is decimal. This decimal chaotic signal can be converted to binary sequence using the threshold comparison approach. Suppose $C_{n}$ is the binary code after quantization, $x_{n}$ is the original decimal chaotic sequence, and $\bar{x}=\lim _{N \rightarrow \infty}(1 / N) \sum_{n=1}^{N} x_{n}$ is the mean of the sequence, which is the threshold. The binary code $C_{n}$ is obtained by the following expression:

$$
\begin{aligned}
C_{n}= & \begin{cases}1, & x_{n} \geq \bar{x} \\
-1, & x_{n}<\bar{x}\end{cases} \\
& n=0,1,2, \ldots .
\end{aligned}
$$

Because there are many parameters in MCPC pulse train signal, the phase code of the subcarrier in each pulse can be changed to obtain MCPC pulse train signals with different properties. Based on the differences of the phase codes of 
each pulse and subcarrier in MCPC pulse train signals, the classification of MCPC pulse train signal is made as shown in Table 1 .

Considering the features of the chaotic sequence mentioned above, we use the chaotic sequence to perform phase code modulation on the MCPC pulse train so as to generate chaotic modulation NNS MCPC pulse train signal. Because different initial values can result in chaotic sequences with different properties, all the chaotic sequences mentioned in the following refer to the chaotic biphase sequence with maximum main-to-sidelobe ratio as selected by the principle of autocorrelation maximization [10]. We design the following two chaotic modulated NNS MCPC pulse train signals with $P$ periods, $N$ subcarriers, and $M$ bits through different chaotic modulation methods.

(1) The Chebyshev mapping generates $L=P \times N \times M$ chaotic biphase codes, which are expressed in set form by $\left\{x_{1}, x_{2}, x_{3}, \ldots x_{L}\right\}$. Suppose the phase coded set of MCPC pulse train signal is $\varphi_{n, m, p}$, where $p=1,2, \ldots P, n=$ $1,2, \ldots N$, and $m=1,2, \ldots M$. The phase of each subcarrier in MCPC pulse train is modulated by

$$
\varphi_{n, m, p}=x_{M N(p-1)+M(n-1)+m} .
$$

Therefore, the first type of chaotic modulation NNS MCPC pulse train is obtained and denoted by Chaos_NNS MCPC_I. Figure 2 shows the structure of Chaos_NNS MCPC_I pulse train.

(2) Each time a pulse is transmitted, the Chebyshev mapping generates $L=N \times M$ chaotic biphase codes with different initial values. The codes are expressed in set form by $\left\{x_{1}, x_{2}, x_{3}, \ldots x_{L}\right\}$. Suppose the phase coded set of a single MCPC pulse is $\varphi_{n, m}$, where $n=1,2, \ldots N$ and $m=1,2, \ldots M$. The phase of each subcarrier in the present transmitted pulse is modulated based on

$$
\varphi_{n, m}=x_{M(n-1)+m} .
$$

Therefore, the second type of chaotic modulation NNS MCPC pulse train is obtained and denoted by Chaos_NNS MCPC_II. The structure of Chaos_NNS MCPC_II pulse train is shown as Figure 3.

\section{Derivation of Ambiguity Function}

The ambiguity function is an important tool for analyzing radar signals. The ambiguity $\chi(\tau, v)$ of a signal $f(t)$ is given by

$$
\chi(\tau, v)=\int_{-\infty}^{+\infty} f(t) f^{*}(t+\tau) \exp (j 2 \pi v t) d t
$$

where $\tau$ is the time shift variable and $v$ is the frequency shift. By substituting (2) into (7), the ambiguity function of NNS MCPC pulse train is obtained:

$$
\begin{aligned}
& \chi(\tau, v) \\
& =\int_{-\infty}^{+\infty} f_{T r}(t) f_{T r}^{*}(t+\tau) \exp (j 2 \pi v t) d t \\
& =\int_{-\infty}^{+\infty} \sum_{p=1}^{P} \sum_{n=1}^{N} \sum_{m=1}^{M} w_{n} a_{n, m, p} \\
& \times s\left[t-(m-1) t_{b}-(p-1) T_{r}\right] \\
& \times \exp \left[j 2 \pi(n-1)\left(t-(p-1) T_{r}\right) \Delta f\right] \\
& \times \sum_{p_{1}=1}^{P} \sum_{n_{1}=1}^{N} \sum_{m_{1}=1}^{M} w_{n_{1}}^{*} a_{n_{1}, m_{1}, p_{1}}^{*} \\
& \times s^{*}\left[t+\tau-\left(m_{1}-1\right) t_{b}-\left(p_{1}-1\right) T_{r}\right] \\
& \times \exp \left[-j 2 \pi\left(n_{1}-1\right)\right. \\
& \left.\times\left(t+\tau-(p-1) T_{r}\right) \Delta f\right] \\
& \times \exp (j 2 \pi v t) d t \\
& =\sum_{p=1}^{P} \sum_{n=1}^{N} \sum_{m=1}^{M} \sum_{p_{1}=1}^{P} \sum_{n_{1}=1}^{N} \sum_{m_{1}=1}^{M} w_{n} w_{n_{1}}^{*} a_{n, m, p} a_{n_{1}, m_{1}, p_{1}}^{*} \\
& \times \exp \left\{j 2 \pi T_{r} \Delta f\right. \\
& \times\left[\left(p_{1}-1\right)\left(n_{1}-1\right)\right. \\
& -(p-1)(n-1)]\} \\
& \times \exp \left[-j 2 \pi\left(n_{1}-1\right) \tau \Delta f\right] \\
& \times \int_{-\infty}^{+\infty} \exp \left\{j 2 \pi\left[\left(n-n_{1}\right) \Delta f+v\right] t\right\} \\
& \times s\left[t-(m-1) t_{b}-(p-1) T_{r}\right] \\
& \times s^{*}\left[t+\tau-\left(m_{1}-1\right) t_{b}-\left(p_{1}-1\right) T_{r}\right] d t \text {. }
\end{aligned}
$$

Let $\left(n-n_{1}\right) \Delta f+v=F_{d}, t-(m-1) t_{b}-(p-1) T_{r}=t^{\prime}$, and the integral in (8) can be simplified as

$$
\begin{aligned}
& \int_{-\infty}^{+\infty} \exp \left\{j 2 \pi\left[\left(n-n_{1}\right) \Delta f+v\right] t\right\} \\
& \times s\left[t-(m-1) t_{b}-(p-1) T_{r}\right] \\
& \quad \times s^{*}\left[t+\tau-\left(m_{1}-1\right) t_{b}-\left(p_{1}-1\right) T_{r}\right] d t \\
& =\int_{-\infty}^{+\infty} \exp \left\{j 2 \pi F_{d}\left[t^{\prime}+(m-1) t_{b}+(p-1) T_{r}\right]\right\} \\
& \quad \times s\left(t^{\prime}\right) s^{*}\left[t^{\prime}+\tau+\left(m-m_{1}\right) t_{b}+\left(p-p_{1}\right) T_{r}\right] d t^{\prime} \\
& =\exp \left\{j 2 \pi F_{d}\left[(m-1) t_{b}+(p-1) T_{r}\right]\right\}
\end{aligned}
$$




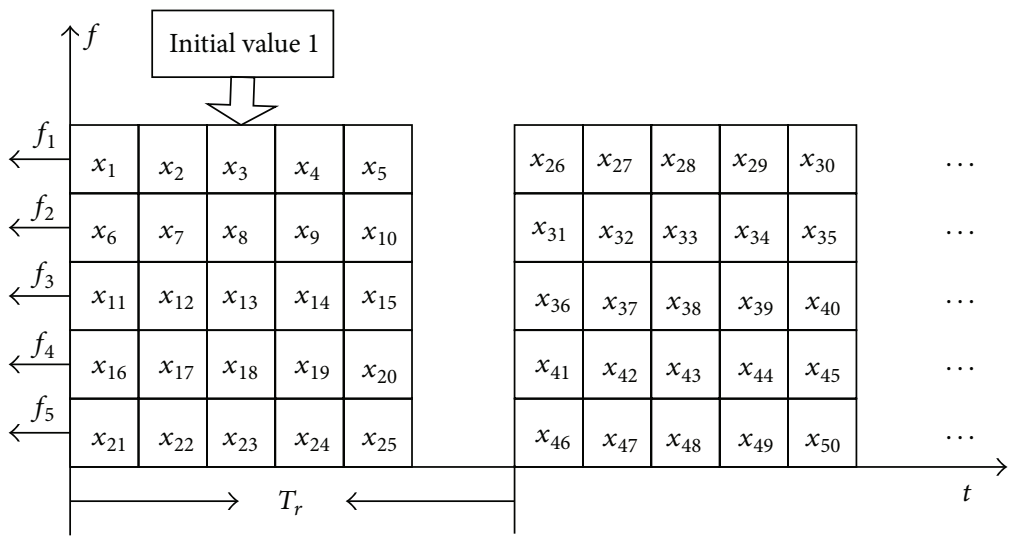

FIGURE 2: Structure of Chaos_NNS MCPC_I pulse train.

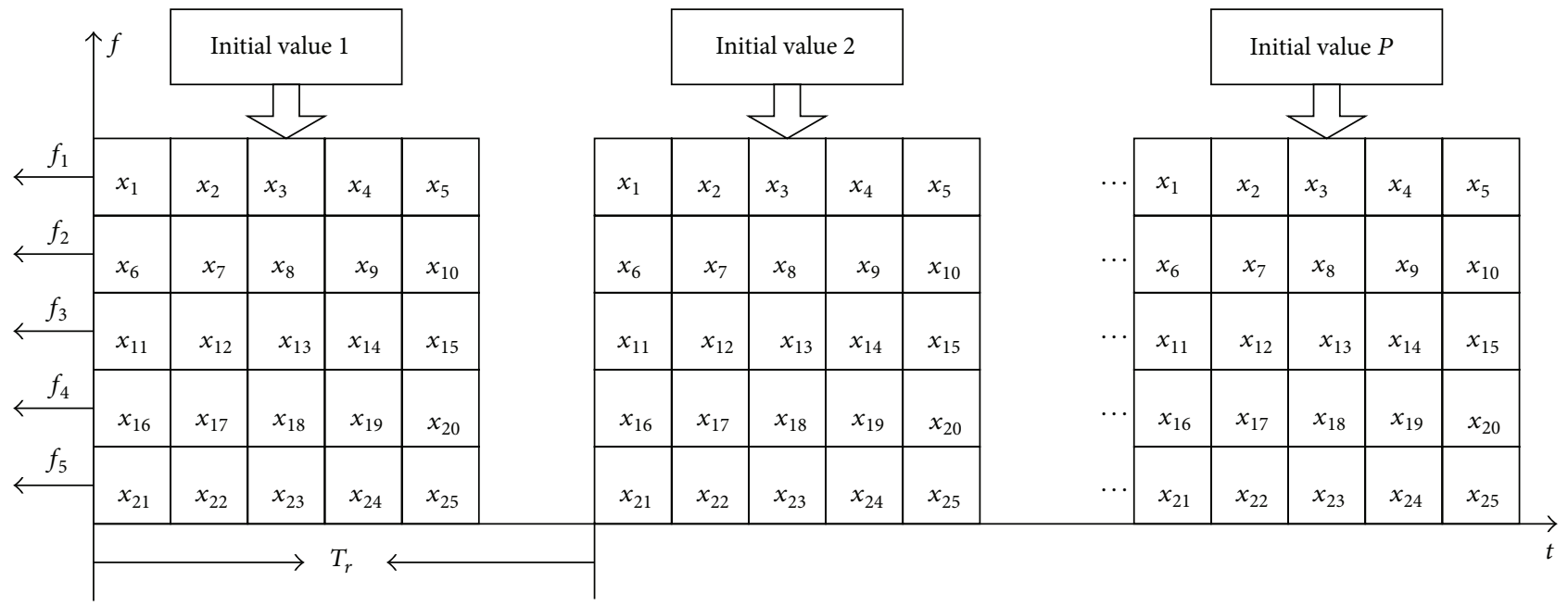

FIgURE 3: Structure of Chaos_NNS MCPC_II pulse train.

$$
\begin{aligned}
\times \int_{-\infty}^{+\infty} \exp \left(j 2 \pi F_{d} t\right) \times s(t) \\
\quad \times s^{*}\left[t+\tau+\left(m-m_{1}\right) t_{b}+\left(p-p_{1}\right) T_{r}\right] d t .
\end{aligned}
$$

Since $s(t) \equiv 1$, for $0 \leq t<t_{b}$, and zero, elsewhere, (8) is nonzero only when $\left|\tau+\left(m-m_{1}\right) t_{b}+\left(p-p_{1}\right) T_{r}\right|<t_{b}$. Let $\tau+\left(m-m_{1}\right) t_{b}+\left(p-p_{1}\right) T_{r}=t_{s}$; then (9) can be abbreviated as

$$
\begin{aligned}
& \exp \left\{j 2 \pi F_{d}\left[(m-1) t_{b}+(p-1) T_{r}\right]\right\} \\
& \quad \times \int_{-\infty}^{+\infty} \exp \left(j 2 \pi F_{d} t\right) \times s(t) s^{*}\left(t+t_{s}\right) d t .
\end{aligned}
$$

If $t_{s}>0$, the integral of (10) becomes

$$
\begin{aligned}
\int_{0}^{t_{b}-t_{s}} \exp \left(j 2 \pi F_{d} t\right) d t & =\left.\frac{e^{j 2 \pi F_{d} t}}{j 2 \pi F_{d}}\right|_{0} ^{t_{b}-t_{s}} \\
& =\frac{e^{j \pi F_{d}\left(t_{b}-t_{s}\right)} \sin \left[\pi F_{d}\left(t_{b}-t_{s}\right)\right]}{\pi F_{d}} ;
\end{aligned}
$$

if $t_{s}<0$, the integral of (10) becomes

$$
\begin{aligned}
\int_{-t_{s}}^{t_{b}} \exp \left(j 2 \pi F_{d} t\right) d t & =\left.\frac{e^{j 2 \pi F_{d} t}}{j 2 \pi F_{d}}\right|_{-t_{s}} ^{t_{b}} \\
& =\frac{e^{j \pi F_{d}\left(t_{b}-t_{s}\right)} \sin \left[\pi F_{d}\left(t_{b}+t_{s}\right)\right]}{\pi F_{d}} .
\end{aligned}
$$

According to (11) and (12), the result for the integral in (10) can be expressed as

$$
\begin{gathered}
\int_{-\infty}^{+\infty} \exp \left(j 2 \pi F_{d} t\right) \times s(t) s^{*}\left(t+t_{s}\right) d t \\
=\frac{e^{j \pi F_{d}\left(t_{b}-t_{s}\right)} \sin \left[\pi F_{d}\left(t_{b}-\left|t_{s}\right|\right)\right]}{\pi F_{d}}, \\
\left|\tau+\left(m-m_{1}\right) t_{b}+\left(p-p_{1}\right) T_{r}\right|<t_{b} .
\end{gathered}
$$


So the ambiguity function of NNS MCPC pulse train is

$$
\begin{aligned}
& \chi(\tau, v) \\
& \begin{aligned}
&=\sum_{p=1}^{P} \sum_{n=1}^{N} \sum_{m=1}^{M} \sum_{p_{1}=1}^{P} \sum_{n_{1}=1}^{N} \sum_{m_{1}=1}^{M} w_{n} w_{n_{1}}^{*} a_{n, m, p} a_{n_{1}, m_{1}, p_{1}}^{*} \\
& \times \exp \left\{j 2 \pi T _ { r } \Delta f \left[\left(p_{1}-1\right)\left(n_{1}-1\right)\right.\right. \\
&-(p-1)(n-1)]\} \\
& \times \exp \left[-j 2 \pi\left(n_{1}-1\right) \tau \Delta f\right] \\
& \times \exp \left\{j 2 \pi F _ { d } \left[(m-1) t_{b}\right.\right. \\
&\left.\left.+(p-1) T_{r}\right]\right\} \\
& \times \frac{e^{j \pi F_{d}\left(t_{b}-t_{s}\right)} \sin \left[\pi F_{d}\left(t_{b}-\left|t_{s}\right|\right)\right]}{\pi F_{d}}, \\
&\left|\tau+\left(m-m_{1}\right) t_{b}+\left(p-p_{1}\right) T_{r}\right|<t_{b} \\
&=\chi_{\text {auto }}(\tau, v)+\chi_{\text {cross }}\left(\tau,\left(n-n_{1}\right) \Delta f+v\right), \\
&\left|\tau+\left(m-m_{1}\right) t_{b}+\left(p-p_{1}\right) T_{r}\right|<t_{b},
\end{aligned}
\end{aligned}
$$

where

$$
\begin{aligned}
& \chi_{\text {auto }}(\tau, v) \\
& \begin{aligned}
=\sum_{\substack{p=1 \\
p=p_{1}} \sum_{n=1}^{N} \sum_{\substack{m=1 \\
m=m_{1}}}^{M}}\left|w_{n}\right|^{2}\left|a_{n, m, p}\right|^{2} \\
\times \exp [-j 2 \pi(n-1) \tau \Delta f] \\
\quad \times \exp \left\{j 2 \pi v\left[(m-1) t_{b}+(p-1) T_{r}\right]\right\} \\
\times \frac{e^{j \pi v\left(t_{b}-t_{s}\right)} \sin \left[\pi v\left(t_{b}-\left|t_{s}\right|\right)\right]}{\pi v}, \quad|\tau|<t_{b},
\end{aligned}
\end{aligned}
$$

is the auto-ambiguity function of Chaos_NNS MCPC pulse train and

$$
\begin{aligned}
& \chi_{\text {cross }}\left(\tau,\left(n-n_{1}\right) \Delta f+v\right) \\
& =\sum_{\substack{p=1 \\
p \neq p_{1}}}^{P} \sum_{\substack{n=1 \\
n \neq n_{1}}}^{N} \sum_{\substack{m=1 \\
m \neq m_{1}}}^{M} \sum_{p_{1}=1}^{P} \sum_{n_{1}=1}^{N} \sum_{m_{1}=1}^{M} w_{n} w_{n_{1}}^{*} a_{n, m, p} a_{n_{1}, m_{1}, p_{1}}^{*} \\
& \times \exp \left\{j 2 \pi T_{r} \Delta f\right. \\
& \times\left[\left(p_{1}-1\right)\left(n_{1}-1\right)\right. \\
& -(p-1)(n-1)]\} \\
& \times \exp \left[-j 2 \pi\left(n_{1}-1\right) \tau \Delta f\right] \\
& \times \exp \left\{j 2 \pi F _ { d } \left[(m-1) t_{b}\right.\right. \\
& \left.\left.+(p-1) T_{r}\right]\right\}
\end{aligned}
$$

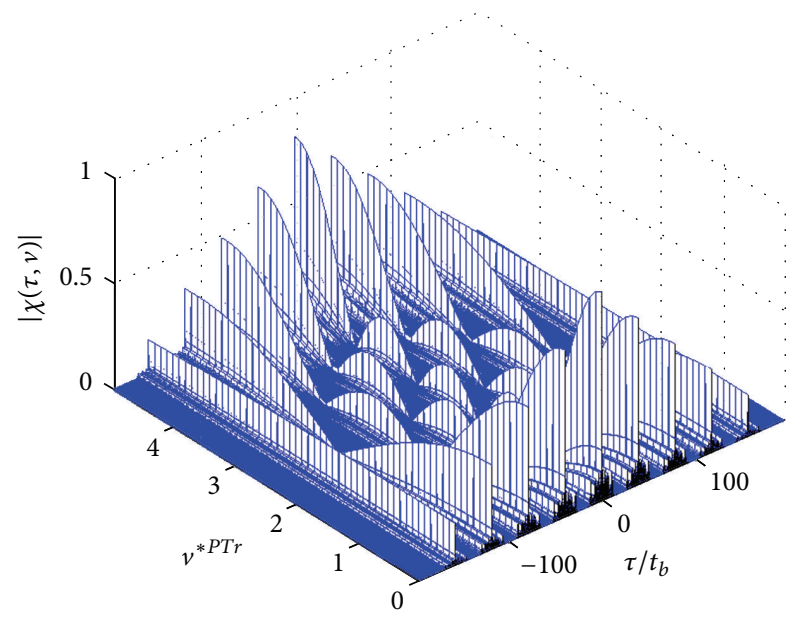

FIgURE 4: Ambiguity function of INS MCPC based on P4 code.

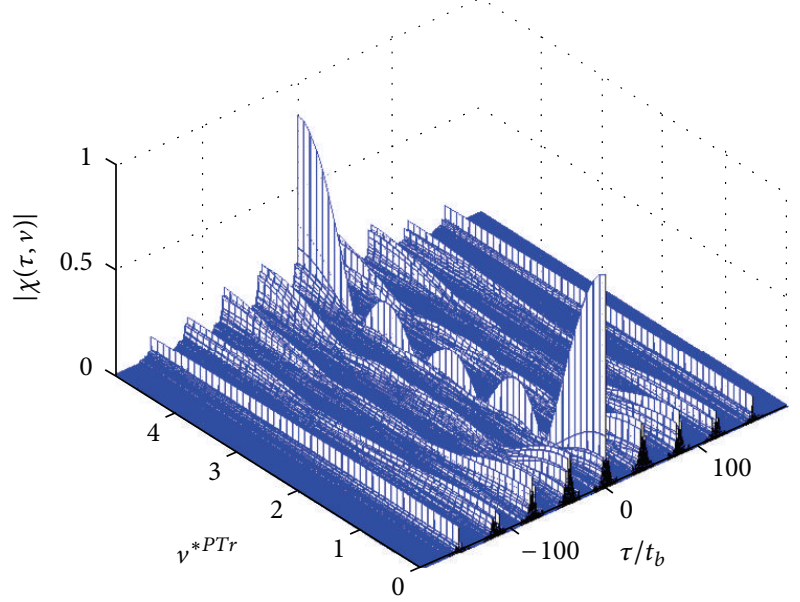

FIgURE 5: Ambiguity function of COCS P4 MCPC pulse train.

$$
\begin{array}{r}
\times \frac{e^{j \pi F_{d}\left(t_{b}-t_{s}\right)} \sin \left[\pi F_{d}\left(t_{b}-\left|t_{s}\right|\right)\right]}{\pi F_{d}}, \\
\left|\tau+\left(m-m_{1}\right) t_{b}+\left(p-p_{1}\right) T_{r}\right|<t_{b},
\end{array}
$$

is the cross-ambiguity function of Chaos_NNS MCPC pulse train.

For Chaos_NNS MCPC_I pulse train, its ambiguity function is (14), where

$$
\begin{aligned}
a_{n, m, p}=e^{j \varphi_{n, m, p}} & =e^{j x_{M N(p-1)+M(n-1)+m}} \\
& =e^{j \cos \left(k \arccos \left(x_{M N(p-1)+M(n-1)+m-1}\right)\right)} .
\end{aligned}
$$

For Chaos_NNS MCPC_II pulse train, its ambiguity function is (14), where

$$
\begin{aligned}
a_{n, m, p}=e^{j \varphi_{n, m, p}} & =e^{j x_{M(n-1)+m}} \\
& =e^{j \cos \left(k \arccos \left(x_{M(n-1)+m-1}\right)\right)} .
\end{aligned}
$$


Furthermore, we note that different $p$ has different initial values in Chaos_NNS MCPC_II pulse train.

\section{Experimental Results}

5.1. Ambiguity Function. The simulation compares the ambiguity of the INS MCPC and NNS MCPC pulse train signals based on P4 code with that of Chaos_NNS MCPC_I and Chaos_NNS MCPC_II proposed in this study. Suppose $P=5$, $N=7, M=13, t_{b}=M \times 10^{-6} \mathrm{~s}$, and the duty ratio is $33 \%$. The phase code of INS MCPC pulse train is the P4 code cyclic shift. Figure 4 shows the ambiguity function of INS MCPC based on $\mathrm{P} 4$ code.

A typical example of NNS MCPC pulse train signal is the COCS (consecutive ordered cyclic shift) P4 code pulse train [2], in which the modulation sequence of all subcarriers of each pulse and all pulses of the same subcarrier are P4 code cyclic shift. Figure 5 shows the ambiguity function of COCS P4 MCPC pulse train.

Since the phase codes in NNS MCPC pulse train signal are different, the periodic side lobe ratio of this signal at integer multiple of $T_{r}$ is obviously lower than that obtained for INS MCPC pulse train signal.

Figure 6 shows the ambiguity function of the Chaos_NNS MCPC_I proposed in this paper with initial values 0.35 .

Figure 7 shows the Chaos_NNS MCPC_II proposed in this paper. The initial values are $[0.75,-0.52,-0.74$, $0.15,0.63]$.

It can be seen from Figures 6 and 7 that the two signals proposed in this paper possess a thumbtack ambiguity function since randomness is introduced into NNS MCPC pulse train signal in each subcarrier and pulse. Thus, it has high range and velocity resolution. The main lobe near the origin is narrow and the periodic side lobe at integer multiple of $T_{r}$ is even lower than that obtained with P4 COCS MCPC pulse train signal. That means the correlation in each period attenuates due to randomness. Although Chaos_NNS MCPC_I and Chaos_NNS MCPC_II pulse train have about the same autocorrelation performance, Chaos_NNS MCPC_II increases the complexity and flexibility of signal design because of different chaotic initial values in each pulse, resulting in higher waveform diversity.

5.2. Analysis on Autocorrelation Performance. There are many parameters in multicarrier chaotic phase code pulse train. Changing the number of subcarriers, bits, and periods will affect the autocorrelation performance of the signal. The simulation result is as follows.

(1) Influence of Subcarrier Number on Autocorrelation. While the numbers of periods and bits are kept unchanged $(P=5$, $M=13)$, the number of subcarriers is increased to three times $(N=21)$ the original number (the sampling rate is also changed to three times the original value). The corresponding autocorrelation performance of Chaos_NNS MCPC_I and Chaos_NNS MCPC_II is shown in Figure 8.

(2) Influence of Bit Number on Autocorrelation. While the numbers of periods and subcarriers are kept unchanged

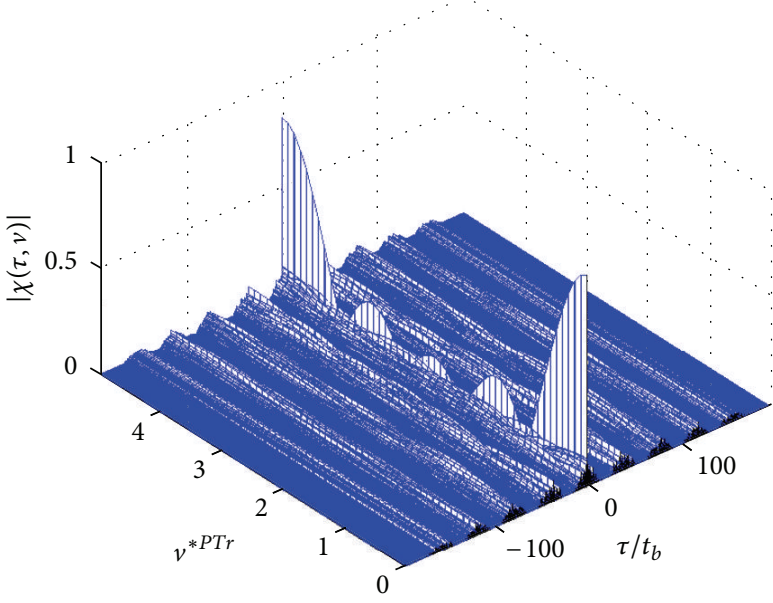

Figure 6: Ambiguity function of Chaos_NNS MCPC_I.

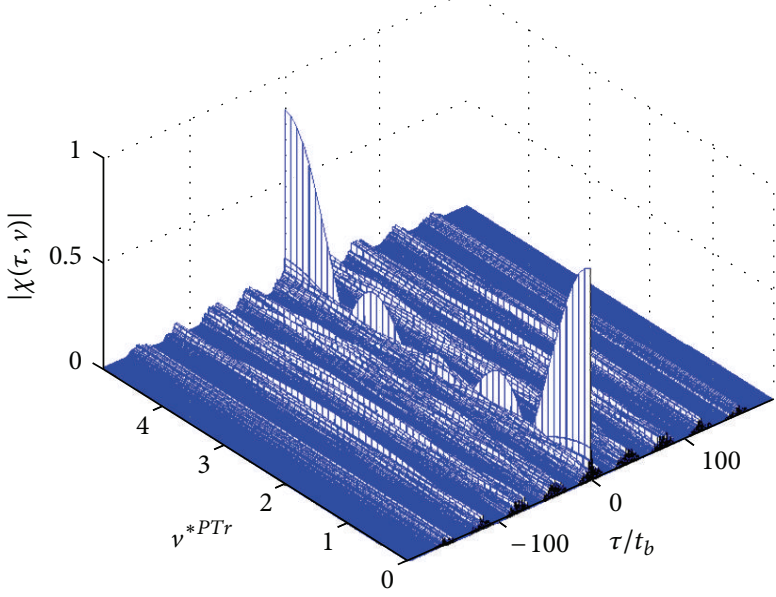

FIGURE 7: Ambiguity function of Chaos_NNS MCPC_II.

( $P=5, N=7)$, the numbers of bits is increased to three times $(M=39)$ the original number. The corresponding autocorrelation performance of Chaos_NNS MCPC_I and Chaos_NNS MCPC_II is shown in Figure 9.

(3) Influence of Period Number on Autocorrelation. While the numbers of subcarriers and bits are kept unchanged $(N=7, M=13)$, the numbers of periods is increased to three times $(P=15)$ the original value. The corresponding autocorrelation performance of Chaos_NNS MCPC_I and Chaos_NNS MCPC_II is shown in Figure 10.

Figures 8 and 9 show that the length of chaotic sequence increases whenever $N$ or $M$ grows. Thus, the randomness increases and the autocorrelation performance improves further. In addition, the autocorrelation of Chaos_NNS MCPC_II is more improved than that of Chaos_NNS MCPC_I. This is because, under the condition of a short chaotic sequence, increasing the length more obviously improves the randomness. It can be seen from Figure 10 that the chaotic sequence becomes longer as the number of periods increases, and the autocorrelation performance 

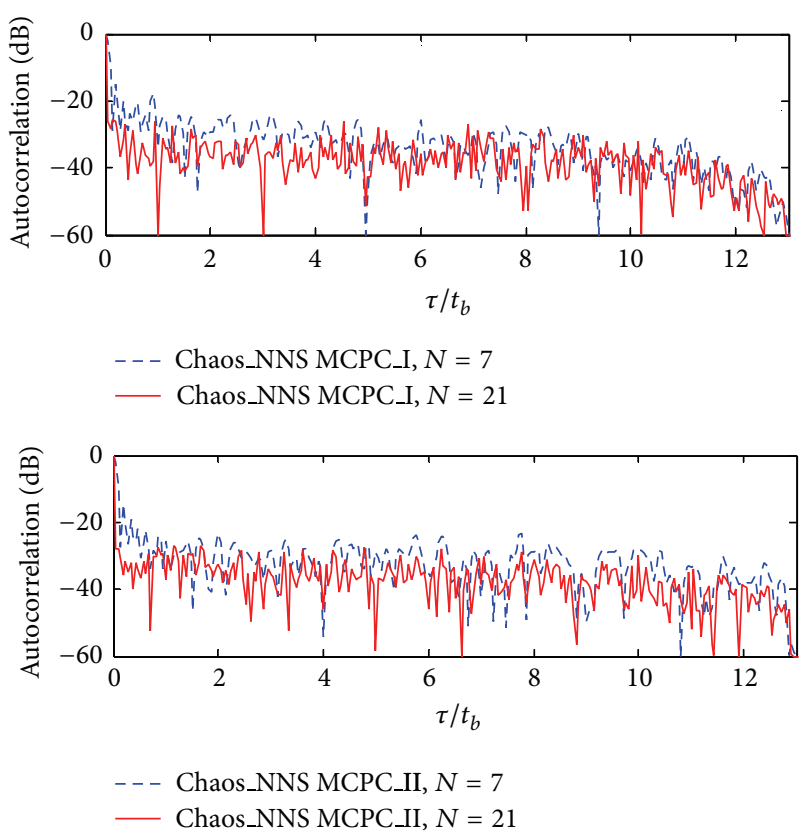

FIGURE 8: Influence of subcarrier number on autocorrelation.

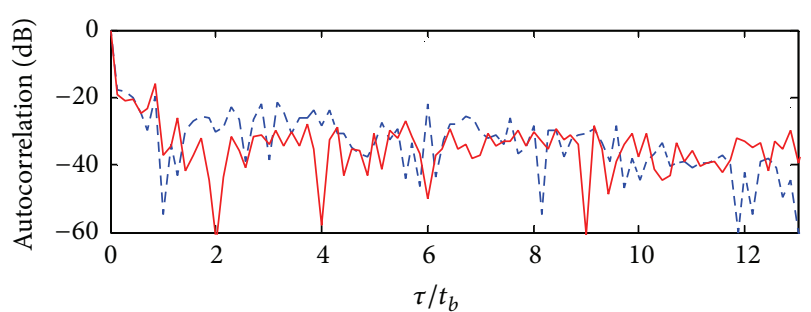

- - - Chaos_NNS MCPC_I, $M=13$

Chaos_NNS MCPC_I, $M=39$

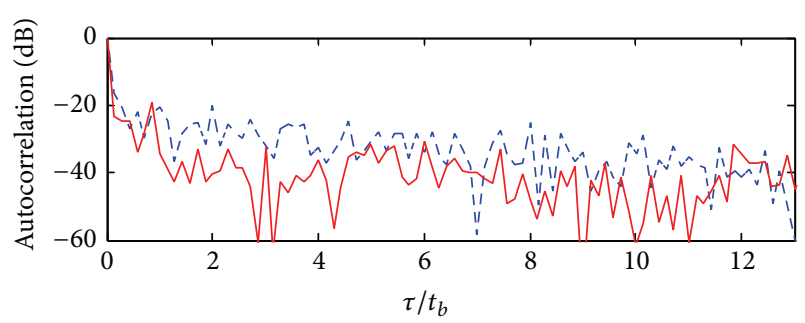

- - - Chaos_NNS MCPC_II, $M=13$

- Chaos_NNS MCPC_II, $M=39$

FIGURE 9: Influence of bit number on autocorrelation.

improves. However, due to the increase of period number, optimal initial values are running out of options, which make the autocorrelation performance of chaotic sequence become worse. Therefore, the improvement of autocorrelation performance of Chaos_NNS MCPC_II signal is not apparent. We can improve the signal autocorrelation performance under large period number by changing the type of chaotic mapping.
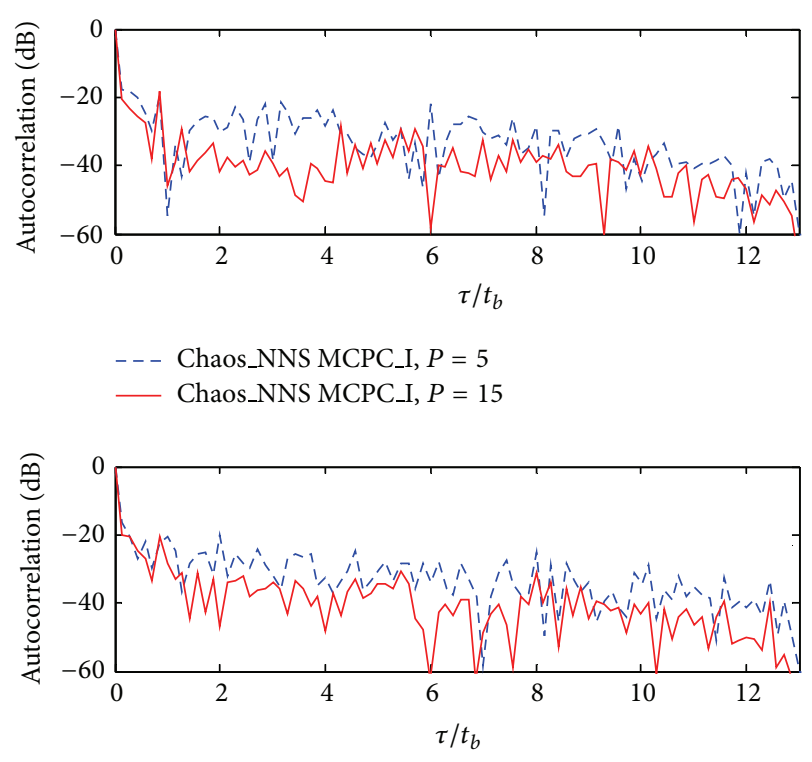

- - Chaos_NNS MCPC_II, $P=5$

- Chaos_NNS MCPC_II, $P=15$

FIGURE 10: Influence of period number on autocorrelation.
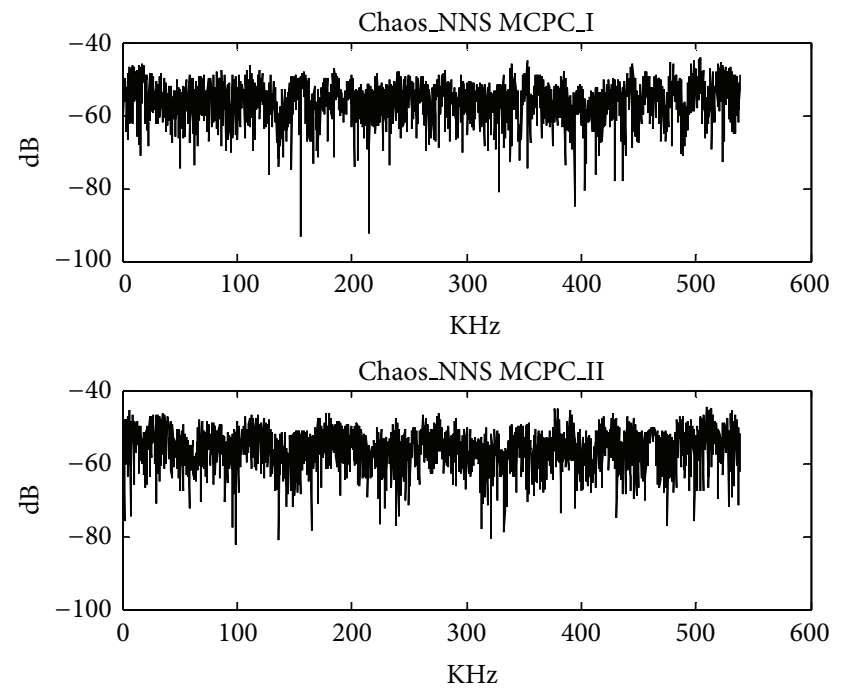

FIgURE 11: Power spectra of Chaos_NNS MCPC_I and Chaos_NNS MCPC_II.

5.3. Analysis on LPI Performance. The intercept factor of radar [11] is written as

$$
\alpha=A \sqrt{\frac{1}{T B}},
$$

where $A$ is a parameter related to radar and reconnaissance receiver and can be regarded as a constant. $T B$ denotes the time-bandwidth product. $T B$ is an important factor that affects the LPI performance of radar. The larger, the better LPI performance is. $B=N / t_{b}$ and $T=M t_{b}$ are the MCPC pulse 
train signal's bandwidth and time width, respectively. So the time-bandwidth product is

$$
T B=N \times M,
$$

and intercept factor is

$$
\alpha=A \sqrt{\frac{1}{N \times M}} .
$$

The LPI performance of MCPC pulse train can be improved by raising the numbers of subcarriers and bits. On the other hand, the power spectra of Chaos_NNS MCPC_I and Chaos_NNS MCPC_II are shown in Figure 11. The figure suggests that the two signals have a flat power spectrum, making it difficult to detect by reconnaissance receivers using the pulse accumulation method [12], which raise the difficulty of being intercepted and identified by enemies. Moreover, because of the randomness of chaos and the complex modulation mode of the signal, the LPI performance is further improved.

\section{Conclusions}

Radar waveform design is one of the main measures to raise the LPI performance of radar and is also an important aspect of radar system design. In this study, the chaotic sequence is introduced into the MCPC pulse train so as to design the Chaos_NNS MCPC_I and Chaos_NNS MCPC_II pulse train signal based on chaotic biphase modulation. We compare the ambiguity function of the signal proposed in this paper with that of MCPC pulse train modulated by P4 code and analyze the influences of the subcarrier number, bit number, and period number on the autocorrelation performance of the two signals. Finally, we discuss the LPI performance of the two signals. The multicarrier chaotic phase coded pulse train signal designed in this paper possesses thumbtack ambiguity function and low periodic autocorrelation side lobe. Also, it has a good LPI performance and the characteristic of waveform diversity, which enhances the detection ability and viability of radar.

\section{Conflict of Interests}

The authors declare that there is no conflict of interests regarding the publication of this paper.

\section{Acknowledgment}

This work was supported by the Aeronautical Science Foundation of Aviation Industry Corporation of China under Grant no. 20112053018.

\section{References}

[1] N. Levanon, "Multifrequency complementary phase-coded radar signal," IEE Proceedings: Radar, Sonar and Navigation, vol. 147, no. 6, pp. 276-284, 2000.

[2] N. Levanon and E. Mozeson, Radar Signals, John Wiley \& Sons, New York, NY, USA, 2004.
[3] D. Bin, Research on the signal designing and processing of multicarrier phase coded radar [Ph.D. thesis], National University of Defense Technology, Changsha, China, 2011, (Chinese).

[4] M. A. Sebt, A. Sheikhi, and M. M. Nayebi, "Orthogonal frequency-division multiplexing radar signal design with optimised ambiguity function and low peak-to-average power ratio," IET Radar, Sonar and Navigation, vol. 3, no. 2, pp. 122$132,2009$.

[5] K. Huo, W.-D. Jiang, X. Li, and J.-J. Mao, "A new OFDM PhaseCoded Stepped-Frequency radar signal and its characteristic," Journal of Electronics and Information Technology, vol. 33, no. 3, pp. 677-683, 2011 (Chinese).

[6] M. I. Skolnik, Introduction to Radar Systems, McGrawHill, 3rd edition, 2003.

[7] S. Boccaletti, C. Grebogi, Y.-C. Lai, H. Mancini, and D. Maza, "The control of chaos: theory and applications," Physics Report, vol. 329, no. 3, pp. 103-197, 2000.

[8] T. L. Carroll, "Adaptive chaotic maps for identification of complex targets," IET Radar, Sonar and Navigation, vol. 2, no. 4, pp. 256-262, 2008.

[9] T. Kohda, A. Tsuneda, and T. Sakae, "Chaotic binary sequences by Chebyshev maps and their correlation properties," in Proceedings of the 2nd IEEE International Symposium on Spread Spectrum Techniques and Applications (ISSTA '92), pp. 63-66, 1992.

[10] J. Lindner, "Binary sequences up to length 40 with best possible autocorrelation function," Electronics Letters, vol. 11, no. 21, p. 507, 1975.

[11] D. C. Schleher, "Low probability of intercept radar," in Proceedings of the International Radar Conference, pp. 346-349, 1985.

[12] P. Babu and P. Stoica, "Spectral analysis of nonuniformly sampled data-a review," Digital Signal Processing, vol. 20, no. 2, pp. 359-378, 2010. 

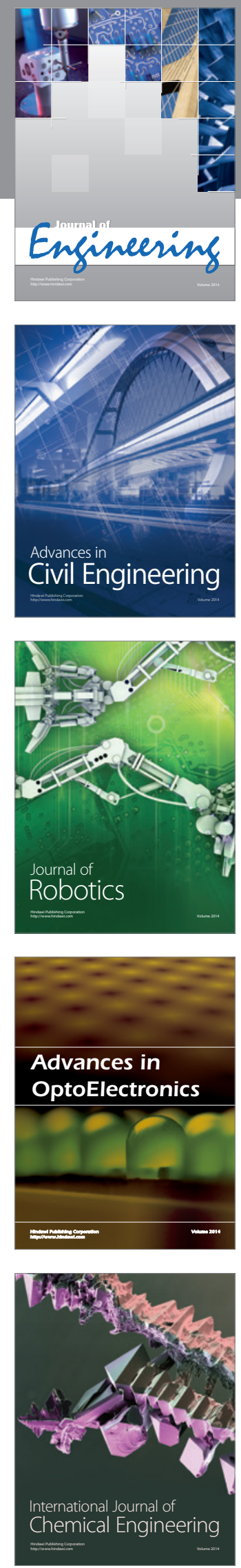

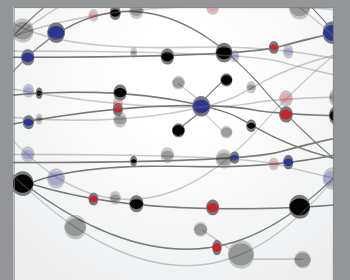

The Scientific World Journal
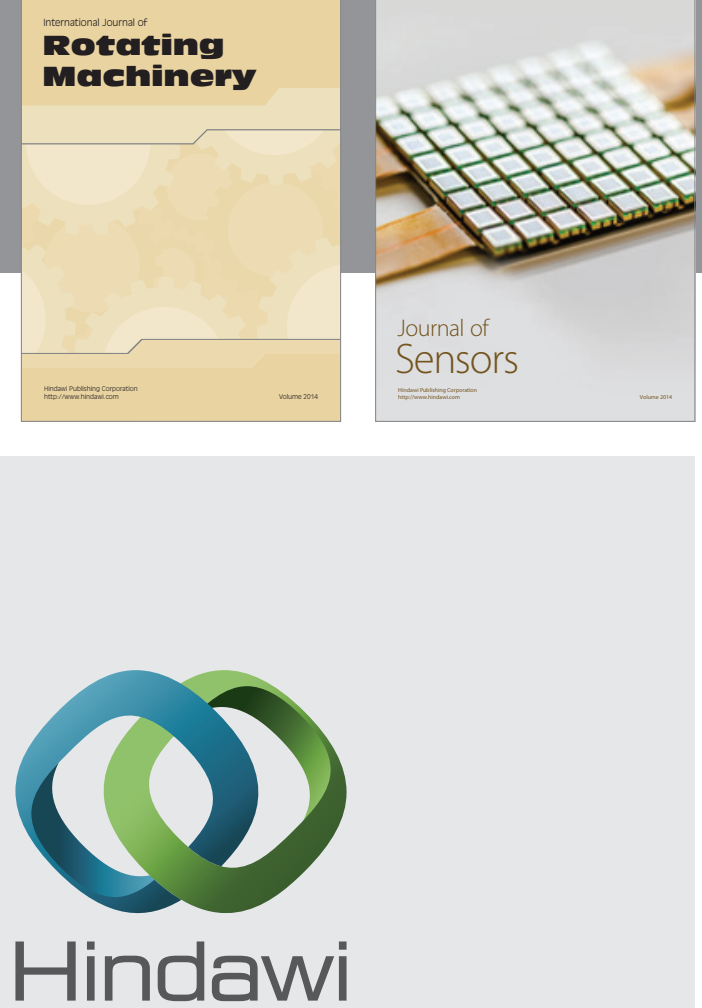

Submit your manuscripts at http://www.hindawi.com
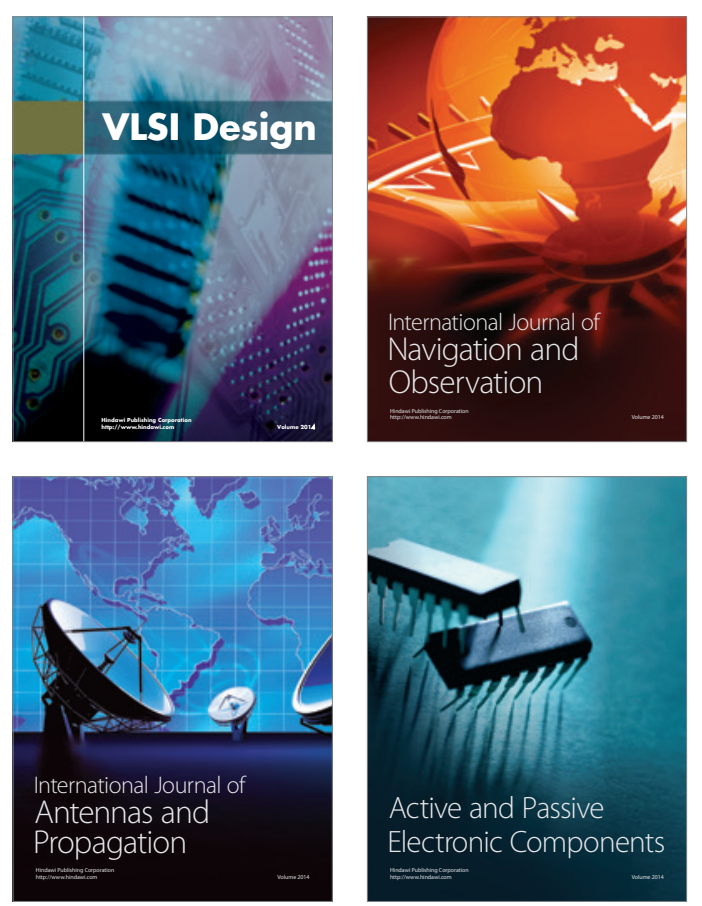
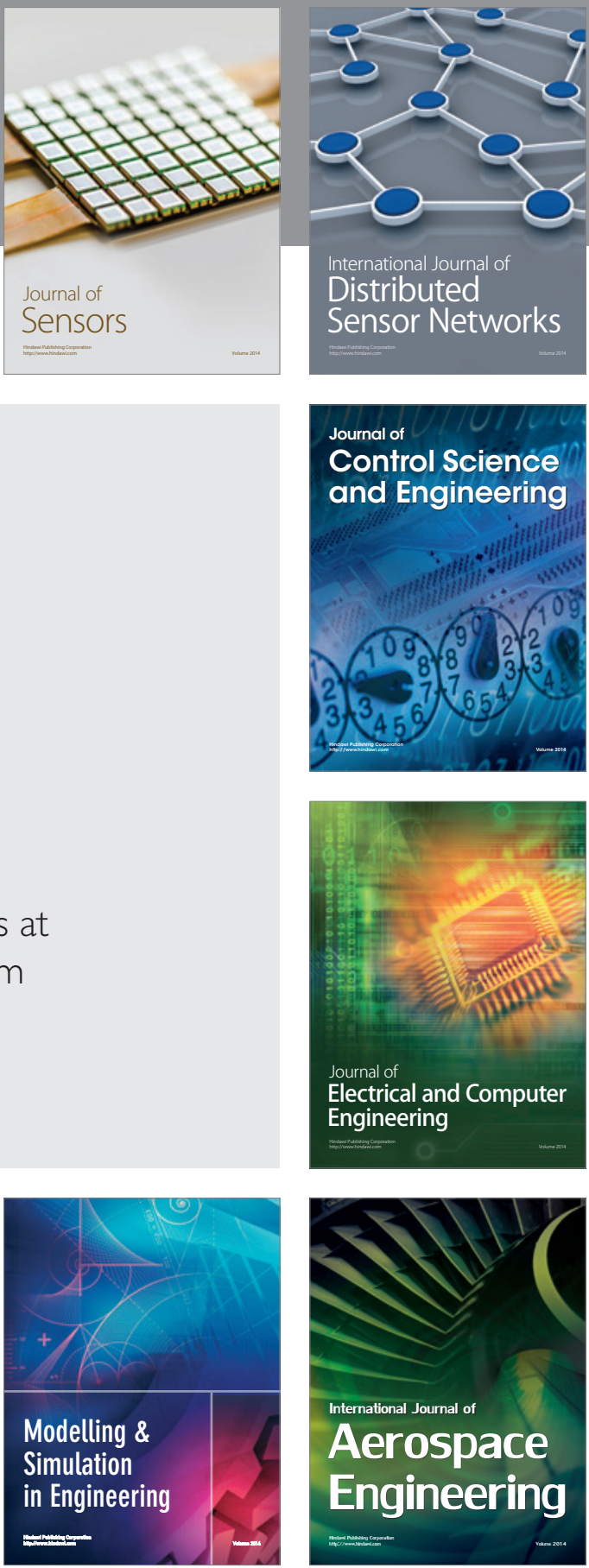

Journal of

Control Science

and Engineering
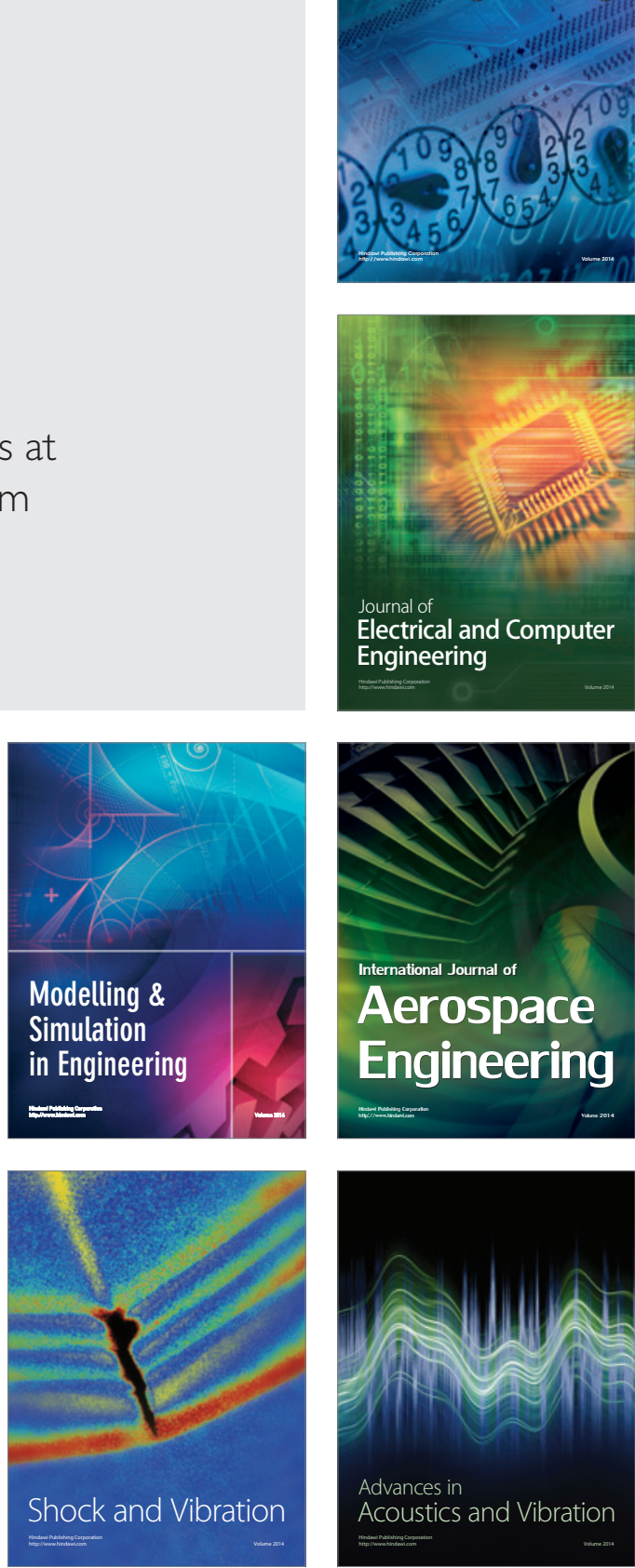